\title{
Medium reach theory for nursing in cardiovascular rehabilitation
}

\author{
Teoria de médio alcance para enfermagem em reabilitação cardiovascular \\ Teoría de rango medio para la enfermería en rehabilitación cardiovascular
}

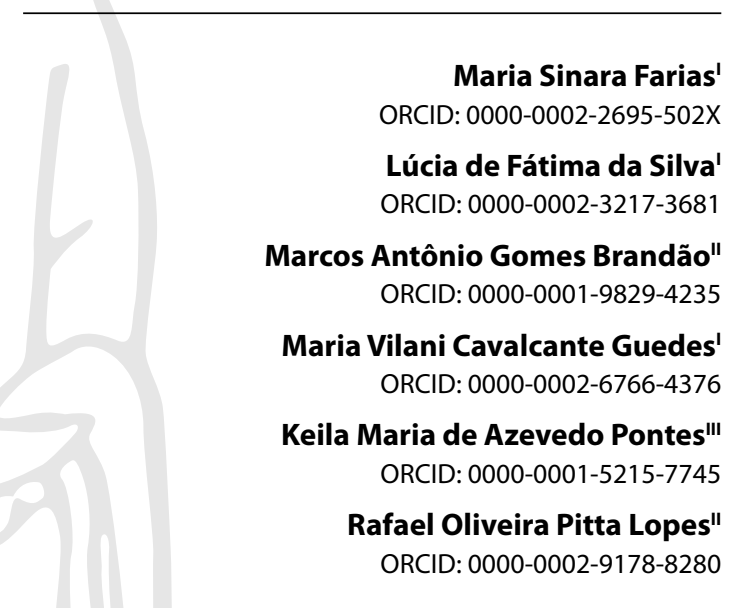

'Universidade Estadual do Ceará. Fortaleza, Ceará, Brazil. "Universidade Federal do Rio de Janeiro. Rio de Janeiro, Rio de Janeiro, Brazil. I'Universidade Estadual Vale do Acaraú. Sobral, Ceará, Brazil.

How to cite this article: Farias MS, Silva LF, Brandão MAG, Guedes MVC, Pontes KMA, Lopes ROP. Medium reach theory for nursing in cardiovascular rehabilitation. Rev Bras Enferm. 2021;74(3):e20190718. https://doi.org/10.1590/0034-7167-2019-0718

\section{Corresponding author:} Maria Sinara Farias E-mail: sinarafariasbc@gmail.com

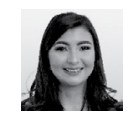

EDITOR IN CHIEF: Antonio José de Almeida Filho ASSOCIATE EDITOR: Maria Elisabete Salvador

Submission: $01-16-2020$

Approval: 01-27-2021

\begin{abstract}
Objectives: to describe a medium-range nursing theory for the cardiovascular rehabilitation process. Methods: theoretical development study, addressing the elaboration of mediumrange theory, based on Roy's Adaptation Model, which combined theoretical deduction with induction strategies, based on literature review and concept analysis. Results: the Medium-Range Theory for Nursing in Cardiovascular Rehabilitation presents eleven concepts and respective definitions, organized in relationships that represent elements, through modeling and image representation. Theoretical assumptions and propositions are presented. Final Considerations: the theory presented aims to contribute to the description of the cardiovascular rehabilitation process from the perspective of the adaptation of the person, family and groups, as well as to impact the nursing care implemented to patients in the process. The procedures advanced from conceptualization to operationalization, through the production of theoretical propositions that will guide future theoretical testing. Descriptors: Nursing Theories; Cardiovascular Diseases; Cardiovascular Rehabilitation; Theoretical Models; Nursing.
\end{abstract}

\section{RESUMO}

Objetivos: descrever uma teoria de enfermagem de médio alcance para o processo de reabilitação cardiovascular. Métodos: estudo de desenvolvimento teórico, orientado para elaboração de teoria de médio alcance, a partir do Modelo de Adaptação de Roy, que combinou estratégias de dedução teórica com indução, a partir de revisão da literatura e análise de conceito. Resultados: a Teoria de Médio Alcance para Enfermagem em Reabilitação Cardiovascular apresenta onze conceitos e respectivas definições, organizados em relações que representam elementos, por meio de modelagem e representação pictórica. Pressupostos e proposições teóricas são apresentadas. Considerações Finais: a teoria apresentada tem como finalidade contribuir para descrição do processo de reabilitação cardiovascular, na perspectiva da adaptação da pessoa, família e grupos, bem como, consequentemente, impactar no cuidado de enfermagem implementado a pacientes no processo. Os procedimentos avançaram da conceitualização até a operacionalização, pela produção de proposições teóricas que guiarão a futura testagem teórica.

Descritores: Teorias de Enfermagem; Doenças Cardiovasculares; Reabilitação Cardiovascular; Modelos Teóricos; Enfermagem.

\section{RESUMEN}

Objetivos: describir una teoría de enfermería de rango medio para el proceso de rehabilitación cardiovascular. Métodos: se trata de un estudio de desarrollo teórico, volcado hacia la elaboración de la teoría de rango medio y basado en el Modelo de Adaptación de Roy que combinó estrategias de inducción y deducción teórica, desde la revisión de la literatura y el análisis del concepto. Resultados: la Teoría de Rango Medio para la Enfermería en Rehabilitación Cardiovascular presenta once conceptos y sus respectivas definiciones, organizados en relaciones que representan elementos, a través de la modelización y la representación pictórica. Se exponen supuestos y propuestas teóricas. Consideraciones Finales: la teoría presentada tiene como intuito contribuir con la descripción del proceso de rehabilitación cardiovascular desde la perspectiva de la adaptación de la persona, la familia y los grupos para causar impacto en los cuidados de enfermería implementados a los pacientes durante el proceso. Los procedimientos avanzaron desde la conceptualización hasta la operacionalización, pasando por la producción de proposiciones teóricas que guiarán las futuras pruebas.

Descriptores: Teorías de Enfermería; Enfermedades Cardiovasculares; Rehabilitación Cardiovascular; Modelos Teóricos; Enfermería. 


\section{INTRODUCTION}

Different countries and regions have proposed programs for the cardiac rehabilitation and secondary prevention process with different approaches ${ }^{(1-6)}$. Despite the relevance of these and other programs available around the world, patient engagement challenges persist, who not always agree to participate, adhere or complete the process as recommended ${ }^{(7)}$.

The use of theories or theoretical models in the elaboration of cardiovascular rehabilitation programs and their results is still an issue under investigation. Systematic review of responses of involvement, adherence and completion of cardiac rehabilitation by patients to interventions based on theories that are not from Nursing did not show significantly changes from the variables linked to patients, even if they could have some result isolated. Among the theories that supported mediations external to the nursing domain, the Theory of Planned Behavior, Social Cognition Theory, Transtheoretical Model of behavior change and Marlatt and Gordon's relapse prevention model(7) are mentioned.

Despite the aforementioned experiences supported by theories that do not come from Nursing, actions of rehabilitation programs are not usually supported by theories, but guided by pragmatics, clinical evidence, clinical guidelines and care lines ${ }^{(8)}$. From the reading of the South American Cardiovascular Prevention and Rehabilitation Guideline, the existence of any theoretical guiding structure to allow the achievement of the rehabilitation results is not easily highlighted, nor has any nursing theory been used $^{(3)}$. In fact, even though the guideline is oriented to fully rehabilitate the patient, with multi-professional practice, there is a physician-centered perspective, whose nursing interventions are: executing the physician's indications, contributing to the patient's education regarding the knowledge of the disease, use of medications and adoption of healthy lifestyle habits, such as exercise practices ${ }^{(3)}$.

Nursing Medium Range Theories (MRT), as guiding structures for cardiovascular rehabilitation, are alternatives that have not yet been proposed, but that may have the potential to generate a more comprehensive practice for human beings and incorporate the singularity of nursing work. Researches that used Great nursing theories for patients with cardiac impairments found sensitive results from the perspective of the profession. A randomized intervention study on the application of an education program based on the Theory of Goal Reaching found favorable changes in cardiovascular risk, in health behaviors and in the quality of life of patients, after the first episode of myocardial infarction ${ }^{(9)}$. Another study examined the effects of a social, educational and exercise support program, based on Roy's Adaptation Model (RAM), verifying that patients in the experimental group adapted better to the condition and the four modes of adaptation ${ }^{(10)}$.

Thus, rehabilitation programs, by incorporating several clinical actions, can benefit from theories of a level closer to practice, such as MRT, which consist of constructions that follow the perspective brought by sociology that locate them between everyday hypotheses and unified theories ${ }^{(11)}$. They seek to overcome the metatheoretical predominance of large or wide-ranging nursing theories, which were developed between the late 1960s and during the 1970s. Later, they were criticized for the scarcity of explicit and testable propositions, as well as for the lack of empirical tests, refutation or theoretical confirmation ${ }^{(12)}$.

Medium-range theories are designed for practical application and have a limited number of testable concepts, variables and relationships. In addition, they offer sufficient generalization for scientific relevance ${ }^{(13)}$. In this context, the development potential of the aforementioned theories is assumed to guide the practice of nurses in cardiovascular rehabilitation (CVR), and subsequent testing in validation in practice.

Therefore, in order to identify a wide-ranging theory to support a nursing MRT for CVR, it is clear that RAM is an essential foundation, in view of its peculiarities. It contributed significantly to the new proposal, as well as by definition of its central concept Adaptation, which comes close to the definition of the studied phenomenon - Cardiovascular Rehabilitation.

In order to contribute to CVR, RAM provides nursing interventions that favor the adaptation of people to assume a lifestyle that provides them well-being. Thus, guidance on the signs and symptoms of the disease, the physical limitations caused by it, with a balanced diet, maintenance of adequate weight and physical activity are important.

Thus, it was assumed that adaptation is a relevant concept for cardiovascular rehabilitation, in the same way Roy's Adaptation Model is a Great theory to be used to deduce a MRT for cardiovascular rehabilitation. Therefore, it is considered the prominent position of Roy's Theory among theories in the field of global nursing, having been applied and tested in different studies since conception ${ }^{(12)}$.

\section{OBJECTIVES}

To describe a medium range nursing theory for the cardiovascular rehabilitation process.

\section{METHODS}

\section{Ethical aspects}

This research, of a theoretical nature, respected the copyright of the publications included in the study. Thus, Law No. 9610, of February 19, 1998 was followed, which alters, updates and consolidates the legislation on copyright and gives other measures ${ }^{(14)}$.

\section{Type of study}

This is a theoretical development study, oriented towards the elaboration of medium-range theory, based on Roy's Adaptation Model (RAM) $)^{(15)}$.

\section{Methodological procedures}

\section{Data collection and analysis}

Theorizing has combined, in an interactive way, the strategies of induction, from literature review, and deduction, through Great nursing theory. This strategy is commonly used in the development of medium-range nursing theories ${ }^{(16)}$.

The general theorizing procedures were: integrative review of literature; analysis of the concept of "cardiovascular rehabilitation", 
with development of the steps by the Walker \& Avant strategy; synthesis of statements obtained from the integrative review; theoretical deduction of concepts and general propositions of the Roy's Adaptation Model; and modeling for model production based on great theory ${ }^{(17)}$.

The integrative review was the technique for searching material for concept analysis. The search incorporated productions in Portuguese, English and Spanish, published since 1966, a mark description of the emergence of Supervised Cardiac Rehabilitation Programs, until April 2018. SciELO, LILACS, BDENF, PUBMED and MEDLINE databases and libraries were included. The descriptors "Cardiovascular Rehabilitation" and "Nursing" were used, connected by the Boolean operator "and". Duplicated studies and those that did not answer the guiding question of the review were excluded: What is the definition of the concept Cardiovascular Rehabilitation?

A total of 42 studies had the necessary elements and were obtained for the analysis of the concept and support in the elaboration of other subsidies of the theory. The information of theoretical interest was: qualitative and quantitative empirical indicators; conceptual and operational definitions; relational statements linked to cardiovascular/cardiac rehabilitation.

The concept analysis involved the steps: determination of goals and purposes, identification of the uses of the concept, determination of attributes, model case identification, identification of other cases, identification of antecedents and consequences, definition of empirical referents ${ }^{(17)}$. The following elements for theorizing were selected: attributes, antecedents, consequents, definitions and representation of a model case, related and contrary to cardiac rehabilitation.

To synthesize statements, generalization procedures of specific inferences were applied, obtained from the reviewed studies, resulting in relational and non-relational statements, of a higher level of abstraction, later being incorporated into the mediumrange theory.

The strategy of theoretical deduction of concepts and general propositions of the Roy's Adaptation Model was carried out after the interpretation of the concepts, propositions, affirmatives, image models of RAM, resulting in more specific elements to the focus of cardiovascular rehabilitation.

The theoretical modeling strategy (for medium range) was the last stage of theorization, which produced a schematic model based on great theory (RAM), resulting in a diagram representative of the elaborated MRT.

\section{RESULTS}

The theoretical development process in applied disciplines involves the conceptualization, operationalization, confirmation and application steps ${ }^{(18)}$. Thus, the results concerning the phases performed are presented: conceptualization and operationalization.

\section{Medium-Range Theory for Nursing in Cardiovascular Rehabilitation}

The conceptualization started from the selection of the concept of cardiovascular rehabilitation that originated the development of the concept analysis strategy. The attributes of the concept included the participation of the patient, family and professionals, the modification of risk factors, behavior interventions, education and counseling practices, and exercise engagements.

By combining the conceptual attributes, induced from the concept analysis, with the deduction of the RAM elements, the definition for the central concept of the theory was elaborated. Thus, in MRT Nur-CVR, cardiovascular rehabilitation is defined as a process that involves the implementation of interventions directed at the person, family and community, through the change of stimulus or strengthening of adaptive processes, being implemented after the cardiovascular event, aiming at physical, social, psychological and spiritual rehabilitation, making the person able to adapt to the new condition.

The antecedents of the concept of cardiovascular rehabilitation were notably linked to risks or diseases, health problems and results of interventions that impaired cardiac capacity, triggering the need for rehabilitation: risk of cardiovascular disease and cardiovascular disease, revascularization surgery, percutaneous coronary interventions, cardiac insufficiency, acute cardiac events, and surgical procedures of the cardiovascular system. In theory, the antecedents facilitated the elaboration of theoretical statements and propositions. The consequences have been aggregated in benefits for the social, physical, psychological and spiritual context of the person, family and other systems related to them.

The antecedents and consequences, as well as the model cases, contrary and limit, provided evidence and empirical representations of cardiovascular rehabilitation, pointing out the approaches and conformations of the studies on the topic addressed. The main function was to anchor the theoretical constructions in the empirical sphere, guaranteeing the characteristic of approximation of theory with practice, essential aspects to medium-range nursing theories.

The concepts selected as relevant for theorizing were: cardiovascular event, adaptation, psychosocial support to the patient and family, health behavior, rehabilitation care, personal care management, educational process in the CVR, cardiac risk, therapy based on physical exercise, supervised cardiovascular rehabilitation program and secondary prevention of DCV.

The affirmative synthesis procedure supported the development of essential definitions for MRT. Eleven constitutive definitions for the concepts are presented:

1. Cardiovascular event is a pathophysiological process that affects the cardiovascular system, which promotes physical, social and psychological changes.

2. Adaptation as a conscious process of choices to respond positively to a cardiac event.

3. Psychosocial support to the patient and family is the care for the patient who suffered the cardiovascular event and their family, with an emphasis on the psychosocial aspects of self-concept, interdependence and role performance.

4. Health behavior is related to the internal and external reactions of the person, family and community to the cardiovascular event, capable of promoting or preventing the integrity/totality of the human adaptive system, configuring states of adaptation of individuals and/or groups. 
These behaviors can be verified before, during and after the occurrence of the cardiovascular event.

5. Rehabilitation nursing care is the implementation of nursing interventions directed at the person, family or community after a cardiovascular event, with a view to physical, social, psychological and spiritual rehabilitation, making it capable of adapting to the new condition.

6. Personal care management is the ability of the person who experienced a cardiovascular event, along with the family and the community, to organize, plan, execute and evaluate actions related to rehabilitation care.

7. Educational process provides health education in all aspects that involve CVR, using guidelines and counseling according to the specific needs of each person, family and community.

8. Cardiac risk is the vulnerability for a person to develop a cardiovascular event.

9. Physical exercise-based therapy is the performance of activities under prescription, supervision and monitoring by a trained professional.

10. Supervised Cardiovascular Rehabilitation Program is a specialized service in which a multi-professional team directs and supervises rehabilitation actions, aimed at people, after a cardiovascular event, considering the phases of evolution.

11. Secondary prevention of cardiovascular disease is the performance of actions to prevent a cardiovascular event, in view of the previous occurrence of a pathophysiological process.

The assumptions were made explicit in the theorization, functioning as ideas accepted and exempt from the need for testing. They proposed us as valid conjectural alternatives for solving the problem underlying the theory. Structured in relation to the metaparadigmatic concepts of nursing, they would presuppose the understanding of the person as adaptive systems, nursing being performed by an active nurse who encourages the person and establishes care actions, also along with other professionals, configuring health from the perspective of the result of an adaptive process.

The propositions were other theoretical elements developed in the operationalization stage. They are described in order to extend the theory in the real world, for creating statements of natural results ${ }^{(18)}$. In the MRT Nur-CVR, a total of seven theoretical propositions were developed:

1. It enables the identification of rehabilitating behaviors and stimulus for the rehabilitation of people who have experienced a cardiovascular event.

2. It enables the establishment of nursing diagnoses, with a view to rehabilitation and planning of rehabilitation care.

3. It involves the implementation of interventions directed at the person after a cardiovascular event, with a view to physical, social and psychological rehabilitation, making them capable of maintaining daily activities.

4. It leads to the management of rehabilitation care by the patient, family and nurse.
5. It involves rehabilitative care, which can be implemented in different environments, including CVR programs, with the use of exercise-based therapy, psychosocial support to the patient and family, and educational processes, helping the patient to achieve readaptation.

6. If rehabilitation care is not implemented, using exercisebased therapy, psychosocial support to the patient and family, and educational processes, the CVR process will not reach its goal and the patient will not be able to rehabilitate efficiently.

7. The reach of the CVR by the patient represents the achievement of the adaptation goal.

It is noteworthy that these proposals will be operationalized through the application of the nursing process based on MRT Nur-CVR, which enables comprehensive and systematic care.

The implementation of the modeling strategy aims to produce a visual representation of the theory under construction ${ }^{(19)}$. The Medium-Range Nursing Theory model organized the concepts and graphically represented the relationships for Cardiovascular Rehabilitation (Figure 1).

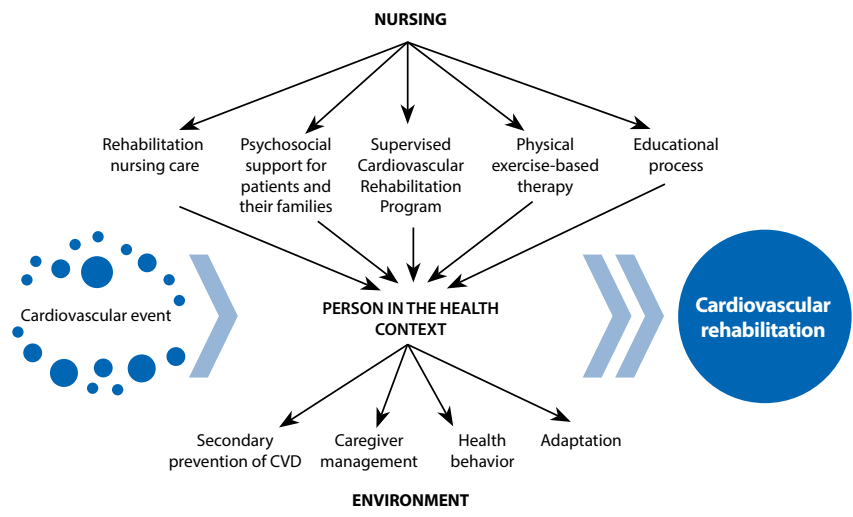

Figure 1 - Medium Range Theory Model for Nursing in Cardiovascular Rehabilitation, Fortaleza, Ceará, Brazil, 2018

\section{DISCUSSION}

The core of the definition of cardiovascular rehabilitation, proposed in MRT Nur-CVR, incorporates the adaptation of the person as a process and result, in which people or groups consciously choose to create human and environmental integration ${ }^{(15)}$. This perspective differs conceptually and expands the idea of rehabilitation based on behavioral, motivational or cognitive-social models external to nursing and which usually have sustained rehabilitation actions ${ }^{(7)}$.

Thus, the deduction of the concept of adaptation brings with it a creative and expanded emphasis for nursing actions. The goal of nursing is to promote the adaptation of individuals and groups in the four adaptive modes, assessing behavior and factors that influence adaptive skills and have improved environmental interactions ${ }^{(15)}$. Therefore, nurses change, increase, decrease, remove and maintain stimulus in such a way that the process of coping with the human adaptive system responds positively.

Usually, cardiovascular rehabilitation is seen from the perspective of the treatment and rehabilitation of the heart and vessels, 
that is, the cardiovascular system ${ }^{(20-21)}$. This view is reductionist and tends to fail in the production of interventions that manage to encompass the complexity of relationships that involve rehabilitation. A study points out the importance of more comprehensive interventions when directing them to exercise, education, counseling for behavioral change and modification of risk factors ${ }^{(22)}$.

Due to the multiprofessional characteristic that the CVR demands, diversified, but converging actions are essential for positive responses to health. Studies show positive effects on the implementation of CVR with regard to symptoms of anxiety, depression, body mass index, physical activity and nutrition ${ }^{(23)}$, such as minimizing social isolation, resulting from financial barriers, and self-management of health ${ }^{(24)}$. However, it is highlighted that nursing depends on a disciplinary perspective to contribute to the projection and articulation of multidisciplinary actions.

Thus, MRT Nur-CVR advances in the coextensive view of the physical and social environments of the individual who experienced the cardiovascular event. It is argued that individuals and groups share creative power, behave intentionally, not in sequence of cause and effect, revealing the existence of an intrinsic holism, in order to maintain integrity and realize the need for relationships $^{(15)}$. This approach, related to the concept of adaptation, has been developed for decades, in a broad way, for knowledge production, research, clinical practice, education programs and administrative systems, especially after the proposition of the Guide for Rehabilitation Nursing Practice ${ }^{(25)}$.

The elaborated MRT incorporated concepts that were judged to be subject to conjecturally appropriate organization, as well as with characteristics that respected the evidence obtained from the concept analysis. It is noteworthy that, in the process of theorization, the selection of appropriate concepts is an important step, both for conceptualization and for future theoretical operation. Concepts must be selected or developed precisely enough to guarantee measurement in operational definitions, or defined in order to maintain semantic clarity ${ }^{(18)}$.

When considering that the identification and definition of concepts can occur in a preliminary way to the definition of the methods to be used for measurement, not all of them must be operational $^{(18)}$, that is, concepts with abstract definitions can be equally important in a theory. However, in MRT Nur-CVR, the clinical nature of the phenomenon tended to lead selection according to concepts aligned with practice. This property allows the definitions produced in the MRT to provide variables that support the construction of measurement instruments, such as scales. Although the definitions can be used for this purpose, it is signaled that, in the set of a theoretical construct, the operational characteristic for testing is provided effectively by the theoretical propositions.

Assumptions have a relevant role in supporting the logical reasoning underlying the choice of constructs and the definition of relationships, and it is opportune to limit them to a small number, when it is a medium-range theory ${ }^{(18-19)}$. When theorizing occurs in qualitative development processes, the assumptions depend on past experiences and will serve as a starting point for theorizing ${ }^{(18)}$, which somehow occurred in the elaboration of the aforementioned theory. In the MRT Nur-CVR, the assumptions considered the authors' experiences, the results of the reviewed studies and the elements of the Roy's Adaptation Model (RAM).
The propositions allow the production of hypotheses that can be tested later ${ }^{(18)}$. For newly created theories, this task is challenging, as is the case with MRT Nur-CVR. The propositions form the interface between conceptualization, which is fundamentally abstract, and operationalization, which provides a path for empirical testing. However, the process of representing practice only reaches more reliable levels in data-based theories. Although the developed MRT used the deduction and conjecture formation process, it also incorporated an inductive integrative review technique, which supported the concept analysis. The combination of induction and deduction can be a facilitator for a practical anchoring of the theory that would facilitate the elaboration of hypotheses derived from the propositions, without abandoning the potential to deduce elements from broader existing theories.

For example, the proposition "CVR involves the implementation of interventions directed at the person, after a cardiovascular event, with a view to physical, social and psychological rehabilitation, making them capable of maintaining daily activities" can be used to investigate the effects of interventions with physical, social, psychological and spiritual coverage on the ability of a person in cardiovascular rehabilitation to maintain activities of daily living at desirable levels. In addition, the proposition "MRT Nur-CVR enables the identification of rehabilitating behaviors and stimulus for the rehabilitation of people who have experienced a cardiovascular event" can be used to design a qualitative study with nurses who have used the theory in practice, in order to verify to what extent, they consider that MRT may have acted in the identification of behaviors and stimulus for rehabilitation.

The MRT Nur-CVR was developed through two aspects of a cardiovascular rehabilitation problem: (1) the absence of an adaptive approach theory, with more comprehensive characteristics that could guide professional care; (2) the need to offer a theoretical framework capable of subsidizing programmatic models and actions, guided by theories, differing from the tendency to establish actions and intervention programs in an empiricist logic and, especially, far from a nursing approach.

Similar to RAM ${ }^{(15)}$, the MRT Nur-CVR model demonstrates the cardiovascular event as the condition that leads the patient to the need to activate coping mechanisms. Thus, Nursing contributes to achieving the goal, in this case, CVR, by manipulating stimulus and behaviors through the implementation of rehabilitative nursing care, promotion of psychosocial support, performance in Supervised Cardiovascular Rehabilitation Programs, with therapies based on exercises associated with educational processes.

In addition, patients need, in context, to assume health behavior and secondary prevention, in addition to managing their own care for adaptation. In this case, the environment-person integration influences the entire CVR process and the results are the behavioral responses of rehabilitation.

From a methodological point of view, the challenges of elaborating medium-range theories are continuous, since, among other factors, it faces the need to establish the appropriate strategies for theorization. It was particularly challenging to develop the synthesis of statements, considering that there was no constant homogeneity in the studies about definitions and conceptualizations. In fact, the responses required by the clinic tend to encourage researchers to overestimate the evidence of practical results 
without maintaining rigor in production and the presentation of constitutive and operational definitions. The consequence of this is that conceptualization becomes more difficult and, at times, more subject to dealing with constructs that may not be fully related to the phenomena investigated in primary research.

It is understood that the limits presented do not detract from the relevance of the constructed theory. Indeed, in shaping this theory and the current state of knowledge on the subject, similar challenges are likely. Nursing theories are sources that support clinical care practices and produce types of (theoretical) evidence that validate certain activities and act to justify, affirm and promote care that intends to be integral and humanized, in the perspective of the elaborated MRT. It is also assumed that, after the theoretical test, the theory can be applied, through the methodological and articulated organization of the Nursing Process, provided for in the conceptualization and operationalization phase.

\section{Limitations of the study}

The MRT Nur-CVR development process was permeated by conceptual and methodological challenges. From a conceptual point of view, there are still limits to the full derivation of the elements of Roy's Adaptation Model ${ }^{(15)}$, especially since the very field of application of the theory, cardiovascular rehabilitation, is marked by studies and practices that are not based on a concept of rehabilitation as an adaptive process, in the integral and totalizing perspective of RAM. Although the theory was developed to circumvent this problem, it becomes influenced by reality, which is not uncommon in applied disciplines, such as Nursing, which must meet both academic and practical standards ${ }^{(18)}$, not allowing theories are purely conjectural.

\section{Contributions to the area of Nursing}

The MRT Nur-CVR intends to contribute to nursing through the novelty in deducing elements of a great nursing theory for the production of a medium-range theory in cardiovascular rehabilitation, indicating an alternative to fundamentally empiricist approaches or guided by theories imported from other disciplines.

In addition, the medium-range conformation facilitates the proposition of practice models that can better accommodate a conception of adaptive processes, a reality to be considered within the scope of theoretical validation.

\section{FINAL CONSIDERATIONS}

This study described a medium-range theory for Nursing in Cardiovascular Rehabilitation that aims to contribute to the description of the cardiovascular rehabilitation process, from the perspective of adapting the person, family and groups, in addition to impacting the implemented nursing care to patients in the process. It was conceptualized, deduced and modeled by applying concept analysis strategies, synthesis of statements, deduction of conceptual model and visual modeling. The procedures advanced from the conceptualization stage to operationalization, by producing theoretical proposals that will guide future theoretical testing.

\section{REFERENCES}

1. Clark RA, Conway A, Poulsen V, Keech W, Tirimacco R, Tideman P. Alternative models of cardiac rehabilitation: a systematic review. Eur J Prev Cardiol. 2015;22(1):35-74. https://doi.org/10.1177/2047487313501093

2. Hamm LF, Sanderson BK, Ades PA, Berra K, Kaminsky LA, Roitman JL, et al. Core competencies for cardiac rehabilitation/secondary prevention professionals. J Cardiopulm Rehabil Prev. 2011;31(1):2-10. https://doi.org/10.1097/HCR.0b013e318203999d

3. Herdy A, López-Jiménez F, Terzic C, Milani M, Stein R, Carvalho T, et al. South American guidelines for cardiovascular disease prevention and rehabilitation. Arq Bras Cardiol. 2014;103(2). https://doi.org/10.5935/abc.2014S003

4. Humphrey R, Guazzi M, Niebauer J. Cardiac Rehabilitation in Europe. Prog Cardiovasc Dis. 2014;56(5):551-6. https://doi.org/10.1016/j. pcad.2013.08.004

5. Song Y. Analyses of studies on cardiac rehabilitation for patients with cardiovascular disease in Korea. J Korean Acad Nurs. 2009;39(3):311. https://doi.org/10.4040/jkan.2009.39.3.311

6. Zhang Z, Pack Q, Squires RW, Lopez-Jimenez F, Yu L, Thomas RJ. Availability and characteristics of cardiac rehabilitation programmes in China. Heart Asia. 2016;8(2):9-12. https://doi.org/10.1136/heartasia-2016-010758

7. Santiago APC, Chaves GS, Davies P, Taylor RS, Grace SL. Interventions to promote patient utilisation of cardiac rehabilitation. Cochrane Database Syst Rev. 2019;(2): CD007131. https://doi.org/10.1002/14651858.CD007131.pub4

8. Timmins F, Horan P. A critical analysis of the potential contribution of Orem's (2001) self-care deficit nursing theory to contemporary coronary care nursing practice. Eur J Cardiovasc Nurs. 2007;6(1):32-9. https://doi.org/10.1016/j.ejcnurse.2006.03.006

9. Park M, Song R, Jeong JO. Effect of goal attainment theory based education program on cardiovascular risks, behavioral modification, and quality of life among patients with first episode of acute myocardial infarction: Randomized study. Int J Nurs Stud. 2017;71: 8-16. https:// doi.org/10.1016/j.ijnurstu.2017.02.019

10. Bakan G, Akyol AD. Theory-guided interventions for adaptation to heart failure. J Adv Nurs. 2008;61(6):596-608. https://doi. org/10.1111/j.1365-2648.2007.04489.x

11. Liehr P, Smith MJ. Middle range theory: a perspective on development and use. Adv Nurs Sci. 2017;40(1):51-63. https://doi.org/10.1097/ ANS.0000000000000162 
12. Im EO,Ju Chang S. Current trends in nursing theories. J Nurs Scholarsh. 2012;44(2):156-64. https://doi.org/10.1111/j.1547-5069.2012.01440.x

13. Fawcett J, DeSanto-Madeya S.Contemporary nursing knowledge: analysis and evaluation of nursing models and theories. 3rd ed. Philadelphia: F.A. Davis Company; 2013

14. Presidência da República (BR). Lei n. 9.610, de 19 de fevereiro de 1998. Altera, atualiza e consolida a legislação sobre direitos autorais e dá outras providências. Diário Oficial da União de Brasília, 19 de fevereiro de 1998.

15. Roy SC. Roy Adaptation Model. 3th ed. Boston: Pearson, Prentice Hall; 2009

16. Brandão MAG, Martins JSA, Peixoto MAP, Lopes ROP, Primo CC. Theoretical and methodological reflections for the construction of middlerange nursing theories. Texto Contexto Enferm. 2017;26(4). https://doi.org/10.1590/0104-07072017001420017

17. Walker LO, Avant KC. Strategies for theory construction in nursing. 5th ed. Boston: Pearson; 2010.

18. Swanson RA,Thomas JC. Theory building in applied disciplines. 15th ed. San Francisco, CA: Berrett-Koehler Publishers; 2013.

19. Weick KE. Theory Construction as Disciplined Imagination. AMR. 1989;14(4):516-531. https://doi.org/10.2307/258556.

20. Grace SL, Bennett S, Ardern Cl, Clark AM. Cardiac Rehabilitation Series: Canada. Prog Cardiovasc Dis. 2014;56(5):530-5. https://doi. org/10.1016/j.pcad.2013.09.010

21. Grossman JAC. Cardiac Rehabilitation Enrollment and the Impact of Systematic Nursing Interventions for Postmyocardial Infarction and Stent Patients. Clin Nurs Res. 2016;25(4):378-90. https://doi.org/10.1177/1054773815620777

22. Feinberg JL, Russell D, Mola A, Trachtenberg M, Bick I, Lipman TH, et al. A Mixed methods evaluation of the feasibility and acceptability of an adapted cardiac rehabilitation program for home care patients. Geriatr Nurs (Minneap). 2018;39(2):191-8. https://doi.org/10.1016/j. gerinurse.2017.08.006

23. Madueño Caro AJ, Mellado Fernández ML, Delgado Pacheco J, Muñoz Ayllon M, Pardos Lafarga M, Saez García L. Autoeficacia percibida, rasgos de personalidad y biotipos previos a programa de rehabilitación cardíaca en atención primaria de salud. Enferm Clín. 2017;27(6):346-51. https://doi.org/10.1016/j.enfcli.2017.04.004

24. Westland H, Bos-Touwen ID, Trappenburg JCA, Schröder CD, de Wit NJ, Schuurmans MJ. Unravelling effectiveness of a nurse-led behavior change intervention to enhance physical activity in patients at risk for cardiovascular disease in primary care: study protocol for a cluster randomized controlled trial. Trials. 2017;18(1):79. https://doi.org/10.1186/s13063-017-1823-9

25. Piazza D, Foote A. Roy's Adaptation Model: a guide for rehabilitation nursing practice. Rehabil Nurs. 1990;15(5):254-9. https://doi. org/10.1002/j.2048-7940.1990.tb01488.x 